\title{
Qualidade de vida de pacientes renais crônicos terminais em uso de terapia renal substitutiva
}

\author{
Quality of life of terminal chronic renal \\ patients using substuctive kidney therapy
}

\section{Marilaine Matos de Menezes Ferreira' ${ }^{1}$ (D) Lara Terezinha Cunha Pereira² (i)}

\author{
${ }^{1}$ Escola Bahiana de Medicina e Saúde Pública (Salvador). Bahia, Brasil. mferreira1@bahiana.edu.br \\ ${ }^{2}$ Autora para correspondência. Escola Bahiana de Medicina e Saúde Pública (Salvador). Bahia, Brasil. laracunha29@gmail.com
}

\begin{abstract}
RESUMO | OBJETIVO: identificar os fatores relacionados às terapias renais substitutivas que interferem na qualidade de vida de pacientes renais crônicos terminais. METODOLOGIA: trata-se de uma revisão integrativa utilizando os descritores "Insuficiência renal crônica", "Transplante de rim", "Diálise renal", "Qualidade de vida" e "Readmissão de paciente", aplicados e combinados de formas diferentes na Biblioteca Virtual de Saúde (BVS) e periódicos CAPES. RESULTADOS: foram encontradas 96 publicações e 16 foram selecionadas, entre 2015 e 2019, de acordo com os critérios estabelecidos. Conforme a predominância do assunto abordado, foram nomeadas categorias temáticas: sentimentos vivenciados de acordo com a escolha da terapia renal substitutiva; expectativas relacionadas ao transplante; transplante renal e a função do enxerto; fatores que interferem na qualidade de vida durante a terapia renal substitutiva. Foram identificados sentimentos, expectativas, facilidades e dificuldades após o transplante renal para o funcionamento do enxerto, além de fatores físicos, psicológicos e assistenciais relacionados à terapia renal substitutiva e como estes interferem na qualidade de vida de pessoas com doença renal terminal. CONCLUSÃo: impactos físicos e psicológicos, além da qualidade da assistência na terapia renal substitutiva interferem na qualidade de vida da pessoa com doença renal crônica em estágio terminal.
\end{abstract}

DESCRITORES: Insuficiência renal crônica. Transplante de rim. Diálise renal. Qualidade de vida.

\begin{abstract}
OBJECTIVE: to identify factors related to renal replacement therapies that interfere in the quality of life of end-stage renal disease patients. METHODOLOGY: this is an integrative review using the descriptors "Chronic renal failure", "Kidney transplantation", "Renal dialysis", "Quality of life" and "Patient readmission", applied and combined in different ways in the Virtual Library (BVS) and CAPES journals. RESULTS: 96 publications were found and 16 were selected, between 2015 and 2019, according to the established criteria. According to the predominance of the subject addressed, thematic categories were named: feelings experienced according to the choice of renal replacement therapy; expectations related to transplantation; kidney transplantation and graft function; factors that interfere with quality of life during renal replacement therapy. Feelings, expectations, facilities and difficulties were identified after kidney transplantation for graft functioning, in addition to physical, psychological and care factors related to renal replacement therapy and how these interfere in the quality of life of people with end-stage renal disease. CONCLUSION: physical and psychological impacts, in addition to the quality of care in renal replacement therapy, interfere with the quality of life of people with endstage chronic kidney disease.
\end{abstract}

KEYWORDS: Chronic renal failure. Kidney transplantation. Renal dialysis. Quality of life. 


\section{Introdução}

A doença renal crônica (DRC), dentre as doenças renais, é uma das mais frequentes e mais graves. Esta consiste em uma lesão renal que persiste por três meses ou mais. Seus estágios são classificados de acordo com a taxa de filtração glomerular e em seu estágio terminal é necessária a adoção de terapias renais substitutivas (TRS) para manutenção da vida, como a hemodiálise, diálise peritoneal ou transplante renal.

De acordo com dados da Sociedade Brasileira de Nefrologia, a doença renal crônica no mundo prevalece $7,2 \%$, para maiores de 30 anos, e $28 \%$ a $46 \%$, para maiores de 64 anos. No Brasil estima-se que cerca de dez milhões de pessoas tenham DRC, dentre elas 90 mil estão em diálise ${ }^{2}$.

A prevalência de pacientes em diálise no Brasil aumentou cerca de $58 \%$ em 2018, quando comparado ao ano de 2009, com um aumento em média de $6,4 \%$ por ano. Com relação ao tipo de diálise, a hemodiálise é a terapia predominante com $92 \%$ dos pacientes, aumento de $3 \%$ em relação a 2009 3

Relacionado à terapia de transplante, houve um aumento de $5,2 \%$ de transplantes renais no Brasil em 2019 comparado a $2012^{4}$. No primeiro trimestre de 2020, também houve um aumento de 3,5\% de transplantes renais em comparação ao ano primeiro trimestre do anterior ${ }^{5}$.

Os portadores da doença renal crônica terminal (DRCT) são muito complexos em seus aspectos físicos e emocionais. Devido à alta prevalência de morbidade e mortalidade, requerem recursos especializados que supram às suas necessidades e os assistam de acordo com o estado de saúde, a situação em que este indivíduo se encontra e como a doença interfere na sua qualidade de vida ${ }^{6}$.

Segundo a Organização Mundial da Saúde (1994), a qualidade de vida refere-se à concepção do indivíduo sobre como ele se encaixa na vida, de acordo com seus valores, cultura, objetivos, expectativas, estilo de vida e preocupações. Isso envolve o bem-estar físico, mental, psicológico, emocional, bem como os relacionamentos sociais, educação, habitação, saneamento básico e a saúde, além de circunstâncias que permeiam à vida ${ }^{?}$.

Os aspectos positivos e negativos relacionados à saúde, bem como a complexidade e dimensão, permitem uma variedade de interpretações e formas de avaliála. Considerando isto, indivíduos com uma mesma enfermidade podem manifestar diferentes condições de saúde e bem-estar, tanto físico quanto emocional. O conceito da qualidade de vida relacionada à saúde (QVRS) baseia-se nessas proposições ${ }^{8}$ e está relacionado à percepção do portador sobre como a doença impacta sobre sua condição de vida útil, considerando as consequências e tratamentos adotados? 9 .

A síndrome urêmica acomete a pessoa à medida que a DRCT avança. Esta síndrome é caracterizada por um conjunto de sinais e sintomas como náuseas, insônia, fadiga, emagrecimento, prurido, comprometimento do estado mental, além de espasmos neuromusculares, fraqueza muscular e câimbras ${ }^{\mathrm{z}}$. As terapias para tratamento da doença renal visam à diminuição desta sintomatologia. O paciente com DRCT tem de conviver com situações advindas da cronicidade da doença e do tratamento, através da TRS, que interfere no estilo de vida do paciente o qual busca lutar pela sobrevida no seu processo de saúde-doença ${ }^{10}$.

A avaliação da qualidade de vida requer uma constante atualização, "devido às possibilidades de mudanças pela temporalidade e na individualidade das pessoas"․․ Essas servem como subsídios para elaboração de estratégias de intervenção para problemas específicos e um suporte especializado que vise diminuir os impactos causados por uma doença de caráter progressivo.

Considerando o que foi exposto, a questão norteadora deste estudo foi: como as terapias renais substitutivas interferem na qualidade de vida de pacientes renais crônicos terminais? Desta forma, o presente estudo buscou identificar os fatores relacionados às terapias renais substitutivas que interferem na qualidade de vida de pacientes renais crônicos terminais, pois interferem diretamente nas medidas a serem adotadas para uma melhor assistência aos portadores. 


\section{Metodologia}

Nesta revisão foram seguidas as etapas propostas para constituição da revisão integrativa de literatura: 1. elaboração de uma questão norteadora e reflexão sobre possíveis hipóteses; 2 . estabelecimento de critérios de inclusão/exclusão para a busca de estudos e seleção da amostra; 3. definição das informações que serão extraídas dos estudos selecionados com apresentação em formas de quadros; 4. avaliação dos estudos com análise crítica dos achados, identificando diferenças ou conflitos; 5. interpretação dos resultados; 6 . apresentar revisão/síntese do conhecimento ${ }^{11}$.

Os artigos elencados foram encontrados a partir da utilização dos Descritores em Ciências da Saúde(DeCS) para pesquisa na Biblioteca Virtual de Saúde (BVS) e periódicos da Coordenação de Aperfeiçoamento de Pessoal de Nível Superior (CAPES) no período de 17 a 20 de abril de 2020.

Os critérios para inclusão dos artigos foram: artigos originais, disponíveis na íntegra eletronicamente, nos idiomas inglês, português ou espanhol, publicados entre 2015 a 2020, envolvendo participantes adultos ( $\geq$ 18 anos) com DRC em estágio terminal e em TRS que relacionem à qualidade de vida. Foram excluídos os estudos que fugiram do escopo temático pretendido, relatos de experiências e revisões de literatura. A faixa etária foi delimitada para melhor direcionamento da pesquisa, considerando que a DRC afeta os grupos etários $<18$ anos e $\geq 18$ anos de formas diferentes ${ }^{12}$.

Foram realizadas duas buscas na BVS para contemplar os assuntos desejados, devido a primeira busca não ter sido suficiente. A primeira busca foi realizada com os seguintes descritores: "Insuficiência renal crônica" and "Transplante de rim" and "Diálise renal" and "Qualidade de vida". Tais descritores foram escoIhidos para melhor contemplar o objetivo do estudo e para explorar as modalidades de terapias renais substitutivas separadamente, justificando a não inclusão do descritor "Terapia de substituição renal" e sim "Transplante de rim" e "Diálise renal".

Foram encontrados 64 artigos dos quais 23 foram selecionados de acordo com a leitura dos resumos.
Posteriormente, feita a leitura completa das publicações, foram selecionados 13 artigos de acordo com os critérios estabelecidos.

A segunda busca na BVS foi realizada com os descritores "Insuficiência renal crônica" and "Transplante de rim" and "Diálise renal" and "Readmissão de paciente". Foram encontrados 3 artigos, dos quais apenas 1 foi selecionado de acordo com os critérios estabelecidos.

Na busca realizada no CAPES foram utilizados os descritores "Transplante de rim" and "Diálise renal", os quais foram escolhidos para melhor obtenção de resultados, visto que as outras combinações realizadas para pesquisa na BVS não apresentaram resultados suficientes. Foram encontrados 29 artigos dos quais 2 foram selecionados de acordo com os critérios estabelecidos.

Foram incluídos no total 16 artigos que se enquadraram dentro da proposta do estudo. Procedeu-se à leitura minuciosa de cada resumo/artigo, destacando aqueles que responderam ao objetivo proposto por este estudo, a fim de organizar e tabular os dados. Para a organização e tabulação dos dados, as pesquisadoras elaboraram instrumentos de coleta de dados contendo: os autores, ano de publicação, país de origem, título do artigo, tipo de estudo e conclusões.

Posteriormente, foram extraídos os conceitos abordados em cada artigo e que responderam à pergunta de investigação. Os trabalhos foram comparados e agrupados por similaridade de conteúdo, sob a forma de categorias empíricas, sendo construídas quatro categorias temáticas, assim especificadas: sentimentos vivenciados de acordo com a escolha da terapia renal substitutiva; expectativas relacionadas ao transplante; transplante renal e a função do enxerto; fatores que interferem na qualidade de vida durante a terapia renal substitutiva.

\section{Resultados e Discussão}

No Quadro 1 estão descritas as variáveis autor, ano de publicação, país de origem, título, tipo de estudo e conclusões dos 16 artigos selecionados. 
Quadro 1. Caracterização dos artigos segundo autor, ano de publicação, país de origem, título, tipo de estudo e conclusões, $n=16$. Salvador, 2020 (continua)

\begin{tabular}{|c|c|c|c|}
\hline $\begin{array}{c}\text { AUTOR/ANO / } \\
\text { PAIS }\end{array}$ & TíTULO & TIPO DO ESTUDO & CONCLUSÕES \\
\hline $\begin{array}{l}\text { Anwar W, Ezzat } \\
\text { H, Mohab A. } \\
2015 \\
\text { Espanha }\end{array}$ & $\begin{array}{l}\text { Comparative study of } \\
\text { impact of hemodialysis } \\
\text { and renal transplantation } \\
\text { on cognitive functions in } \\
\text { ESRD patients. }\end{array}$ & Caso-controle & $\begin{array}{l}\text { O transplante renal como modalidade de } \\
\text { tratamento, em pacientes com DRT, é } \\
\text { superior à hemodiálise em termos de } \\
\text { melhoria do desempenho cognitivo. }\end{array}$ \\
\hline $\begin{array}{l}\text { Andrade SV, Sesso } \\
\text { R, Diniz DHMP. } \\
2015 \\
\text { Brasil }\end{array}$ & $\begin{array}{l}\text { Desesperança, ideação } \\
\text { suicida e depressão em } \\
\text { pacientes renais crônicos } \\
\text { no tratamento por } \\
\text { hemodiálise ou } \\
\text { transplante. }\end{array}$ & $\begin{array}{l}\text { Observacional } \\
\text { transversal }\end{array}$ & $\begin{array}{l}\text { A prevalência de ideação suicida e sintomas } \\
\text { depressivos, na diálise renal e transplante } \\
\text { renal, merece atenção e indica a } \\
\text { necessidade de monitorização e cuidados } \\
\text { nesses pacientes. }\end{array}$ \\
\hline $\begin{array}{l}\text { Salter ML, Kumar } \\
\text { K, Law AH, Gupta } \\
\text { N, Marks K, } \\
\text { Balhara K, et al. } \\
2015 \\
\text { Estados Unidos }\end{array}$ & $\begin{array}{l}\text { Perceptions about } \\
\text { hemodialysis and } \\
\text { transplantation among } \\
\text { African American adults } \\
\text { with end-stage renal } \\
\text { disease: inferences from } \\
\text { focus groups. }\end{array}$ & $\begin{array}{l}\text { Observacional } \\
\text { transversal }\end{array}$ & $\begin{array}{l}\text { Temas: saúde atual e percepções da diálise; } \\
\text { apoio durante a diálise; interações com } \\
\text { profissionais da área médica; preocupações } \\
\text { com o transplante. }\end{array}$ \\
\hline $\begin{array}{l}\text { Santos BP, Viegas } \\
\text { AC, Feijó AM, Lise } \\
\text { F, Schwartz E. } \\
2016 \\
\text { Brasil }\end{array}$ & $\begin{array}{l}\text { Foi/não foi tudo o que } \\
\text { pensava: facilidades e } \\
\text { dificuldades após o } \\
\text { transplante renal. }\end{array}$ & $\begin{array}{l}\text { Descritivo } \\
\text { transversal }\end{array}$ & $\begin{array}{l}\text { As facilidades e as dificuldades dependem } \\
\text { da vivência de cada pessoa. Os profissionais } \\
\text { da saúde necessitam entender e promover } \\
\text { ações de saúde que favoreçam a } \\
\text { singularidade e o contexto do } \\
\text { transplantado renal. }\end{array}$ \\
\hline $\begin{array}{l}\text { Von der Lippe N, } \\
\text { Waldum-Grevbo B, } \\
\text { VarbergReisæter A, } \\
\text { Os I. } \\
2016 \\
\text { Noruega }\end{array}$ & $\begin{array}{l}\text { Is HRQOL in dialysis } \\
\text { associated with patient } \\
\text { survival or graft function } \\
\text { after kidney } \\
\text { transplantation?. }\end{array}$ & $\begin{array}{l}\text { Prospectivo } \\
\text { Iongitudinal }\end{array}$ & $\begin{array}{l}\text { A má função física medida durante a diálise } \\
\text { foi associada ao aumento da mortalidade } \\
\text { até sete anos após o transplante e } \\
\text { problemas de saúde mental em pacientes } \\
\text { em diálise estavam associados ao declínio } \\
\text { acelerado da função do enxerto após o } \\
\text { transplante. }\end{array}$ \\
\hline $\begin{array}{l}\text { Pauletto MR, } \\
\text { Beuter M, Timm } \\
\text { AMB, Santos NO, } \\
\text { Roso CC, Jacobi CS. } \\
2016 \\
\text { Brasil } \\
\end{array}$ & $\begin{array}{l}\text { Transplante renal: } \\
\text { percepção de pacientes } \\
\text { em hemodiálise fora da } \\
\text { lista de espera. }\end{array}$ & $\begin{array}{l}\text { Descritivo } \\
\text { exploratório }\end{array}$ & $\begin{array}{l}\text { As percepções dos pacientes sobre o } \\
\text { transplante influenciam a decisão de não } \\
\text { ingressarem em lista, por implicar em } \\
\text { mudanças e limitações na vida diária, para } \\
\text { as quais ainda não estão preparados. }\end{array}$ \\
\hline $\begin{array}{l}\text { Lai C, Aceto P, } \\
\text { Luciani M, Fazzari } \\
\text { E, Cesari V, Luciano } \\
\text { S, et al. } \\
2017 \\
\text { Italia }\end{array}$ & $\begin{array}{l}\text { Emotional management } \\
\text { and biological markers of } \\
\text { dietetic regimen in chronic } \\
\text { kidney disease patients. }\end{array}$ & $\begin{array}{l}\text { Observacional } \\
\text { transversal }\end{array}$ & $\begin{array}{l}\text { A diálise peritoneal pode diminuir o bem- } \\
\text { estar psicológico e a adesão ao tratamento } \\
\text { na DRC em estágio final. }\end{array}$ \\
\hline $\begin{array}{l}\text { Brekke FB, } \\
\text { Waldum-Grevbo B, } \\
\text { Von der Lippe N, } \\
\text { Os I. } \\
2017 \\
\text { Noruega }\end{array}$ & $\begin{array}{l}\text { The effect of renal } \\
\text { transplantation on quality } \\
\text { of sleep in former dialysis } \\
\text { patients. }\end{array}$ & $\begin{array}{l}\text { Observacional } \\
\text { Iongitudinal }\end{array}$ & $\begin{array}{l}\text { O transplante pode melhorar a qualidade } \\
\text { do sono em quase metade dos pacientes, } \\
\text { mas um grande número de pacientes ainda } \\
\text { sofria de distúrbios do sono após o } \\
\text { transplante. }\end{array}$ \\
\hline $\begin{array}{l}\text { Haugen CE, King } \\
\text { EA, Bae S, Bowring } \\
\text { MG, Holscher CM, } \\
\text { Garonzik-Wang J, et } \\
\text { al. } \\
2018 \\
\text { Estados Unidos }\end{array}$ & $\begin{array}{l}\text { Early Hospital Readmission } \\
\text { in Older and Younger } \\
\text { Kidney Transplant } \\
\text { Recipients. }\end{array}$ & $\begin{array}{l}\text { Observacional } \\
\text { longitudinal }\end{array}$ & $\begin{array}{l}\text { Os receptores mais velhos de transplante } \\
\text { renal têm maior probabilidade de } \\
\text { apresentar readmissão hospitalar precoce e } \\
\text { correm maior risco de perda de enxerto } \\
\text { após a readmissão do que os mais jovens. }\end{array}$ \\
\hline
\end{tabular}




\begin{tabular}{|c|c|c|c|}
\hline $\begin{array}{l}\text { AUTOR/ANO / } \\
\text { PAIS }\end{array}$ & TíTULO & TIPO DO ESTUDO & CONCLUSÕES \\
\hline $\begin{array}{l}\text { Freitas MHB, Lima } \\
\text { LC, Couceiro TCM, } \\
\text { Silva WB, Andrade } \\
\text { JM, Freitas MHB. } \\
2018 \\
\text { Brasil } \\
\end{array}$ & $\begin{array}{l}\text { Fatores perioperatórios } \\
\text { associados à função tardia } \\
\text { do enxerto em pacientes } \\
\text { transplantados renais. }\end{array}$ & Coorte histórica & $\begin{array}{l}\text { Técnica combinada de anestesia, diálise por } \\
\text { mais de } 60 \text { meses, Basiliximab e tempo de } \\
\text { isquemia fria> } 12 \text { horas são fatores de risco } \\
\text { para função tardia do enxerto; regimes de } \\
\text { fluidos liberais e rins de doadores vivos são } \\
\text { protetores. }\end{array}$ \\
\hline $\begin{array}{l}\text { Santos LF, Prado } \\
\text { BC, Castro FPS, } \\
\text { Brito R, Maciel SC, } \\
\text { Avelar TC. } \\
2018 \\
\text { Brasil } \\
\end{array}$ & $\begin{array}{l}\text { Qualidade de Vida em } \\
\text { Transplantados Renais. }\end{array}$ & $\begin{array}{l}\text { Descritivo } \\
\text { transversal }\end{array}$ & $\begin{array}{l}\text { O transplante favoreceu a melhoria da } \\
\text { qualidade de vida dos pacientes e nortear } \\
\text { os profissionais de saúde. }\end{array}$ \\
\hline $\begin{array}{l}\text { Grove BE, } \\
\text { Schougaard LM, } \\
\text { Hjollund NH, } \\
\text { Ivarsen P. } \\
2018 \\
\text { Dinamarca }\end{array}$ & $\begin{array}{l}\text { Self-rated health, quality of } \\
\text { life and appetite as } \\
\text { predictors of initiation of } \\
\text { dialysis and mortality in } \\
\text { patients with chronic } \\
\text { kidney disease stages 4-5: } \\
\text { a prospective cohort } \\
\text { study. }\end{array}$ & $\begin{array}{l}\text { Coorte } \\
\text { prospectivo }\end{array}$ & $\begin{array}{l}\text { A autoavaliação de saúde em pacientes em } \\
\text { tratamento de diálise pode ser melhor que } \\
\text { em pacientes ainda não dialíticos, devido } \\
\text { aos sintomas relacionados à progressão da } \\
\text { doença, os quais são aliviados pela terapia } \\
\text { dialítica. }\end{array}$ \\
\hline $\begin{array}{l}\text { Farias MS, Maia } \\
\text { ICG, Ferreira GSM, } \\
\text { Pinto JR, Ferreira } \\
\text { FIS. } \\
2018 \\
\text { Brasil }\end{array}$ & $\begin{array}{l}\text { Sentimentos de Pessoas } \\
\text { em Hemodiálise que } \\
\text { Esperam por um } \\
\text { Transplante Renal / } \\
\text { Feelings of People } \\
\text { undergoing Hemodialysis } \\
\text { in the Wait for Kidney } \\
\text { Transplantation. }\end{array}$ & $\begin{array}{l}\text { Descritivo } \\
\text { exploratório }\end{array}$ & $\begin{array}{l}\text { Os pacientes que realizam hemodiálise e } \\
\text { que esperam por um transplante renal são } \\
\text { cercados por sentimentos e expectativas } \\
\text { positivas da realização de um transplante } \\
\text { bem sucedido e medos relacionados ao } \\
\text { tratamento e da morte. }\end{array}$ \\
\hline $\begin{array}{l}\text { Lønning K, } \\
\text { Midtvedt K, Heldal } \\
\text { K, Andersen MH. } \\
2018 \\
\text { Noruega }\end{array}$ & $\begin{array}{l}\text { Older kidney } \\
\text { transplantation } \\
\text { candidates' expectations } \\
\text { of improvement in life and } \\
\text { health following kidney } \\
\text { transplantation: } \\
\text { semistructured interviews } \\
\text { with enlisted dialysis } \\
\text { patients aged } 65 \text { years and } \\
\text { older. }\end{array}$ & $\begin{array}{l}\text { Observacional } \\
\text { transversal }\end{array}$ & $\begin{array}{l}\text { Os candidatos mais velhos ao transplante } \\
\text { renal compreendem um grupo } \\
\text { heterogêneo de pacientes que adotam } \\
\text { abordagens individuais que lhes permitem } \\
\text { manter autonomia e controle enquanto } \\
\text { aguardam o transplante. }\end{array}$ \\
\hline $\begin{array}{l}\text { Ramírez CA. } \\
2019 \\
\text { Colômbia }\end{array}$ & $\begin{array}{l}\text { Aprender a vivir con un } \\
\text { órgano trasplantado: } \\
\text { Learning to live with a } \\
\text { transplanted organ. }\end{array}$ & $\begin{array}{l}\text { Observacional } \\
\text { transversal }\end{array}$ & $\begin{array}{l}\text { A doação é um presente que é esperado } \\
\text { pacientemente, com a finalidade de } \\
\text { encerrar um tratamento que gera medo e } \\
\text { desesperação. Esta alternativa de } \\
\text { tratamento leva aos pacientes a terem a } \\
\text { sensação de renascença e de recuperação } \\
\text { da vida que foi perdida pela diálise, embora } \\
\text { reconheçam que é finito. }\end{array}$ \\
\hline $\begin{array}{l}\text { Brito DCS, } \\
\text { Machado EL, Reis } \\
\text { IA, Carmo LPF, } \\
\text { Cherchiglia ML. } \\
2019 \\
\text { Brasil }\end{array}$ & $\begin{array}{l}\text { Depression and anxiety } \\
\text { among patients } \\
\text { undergoing dialysis and } \\
\text { kidney transplantation: a } \\
\text { cross-sectional study. }\end{array}$ & $\begin{array}{l}\text { Observacional } \\
\text { transversal }\end{array}$ & $\begin{array}{l}\text { Os sintomas de depressão e ansiedade } \\
\text { ocorreram com maior frequência entre os } \\
\text { pacientes em diálise. Qualidade de vida, } \\
\text { comorbidades e perda de acesso vascular } \\
\text { foram fatores associados. }\end{array}$ \\
\hline
\end{tabular}


Dentre os artigos selecionados, publicações do ano de 2018 foram as mais encontradas (6/16), seguidos pelos anos de 2015 (3/16), 2016 (3/16), 2017 (2/16) e 2019 (2/16). Com relação ao país de origem do estudo, o Brasil se destacou com o maior número de publicações (7/16), seguido dos países Noruega (3/16), Estados Unidos (2/16), Colômbia, Itália e Dinamarca só apresentaram uma publicação cada (1/16).

Os cenários de pesquisa variaram entre clínicas de hemodiálise, ambulatórios especializados em nefrologia, hospitais e centros de diálise. A abordagem quantitativa foi a mais frequente dentre os estudos, comparada com abordagem qualitativa, embora represente (9/16) dos achados.

A maioria dos estudos apresenta como tipo de delineamento o observacional/descritivo transversal (8/16), seguidos de observacionais/prospectivos longitudinais (3/16), descritivos exploratórios (2/16) e estudos de coorte (2/16), e estudo caso-controle (1/16).

A coleta de dados com aplicação de questionários foi a mais frequente, mas também foram utilizadas coletas em bancos de dados, prontuários, testes e exames clínicos, exames laboratoriais, discussões em grupos focais e entrevistas estruturadas e semiestruturadas. Com relação às pesquisas qualitativas, a entrevista semiestruturada foi o método mais utilizado.

Conforme a predominância do assunto abordado na análise dos artigos, foram nomeadas as categorias temáticas abaixo:

\section{Sentimentos vivenciados de acordo com a escolha da terapia renal substitutiva}

As pessoas acometidas pela doença renal terminal e consequentemente submetidas às terapias de substituição, como a diálise e transplante renal, vivenciam diversas emoções durante o curso e tratamento da doença ${ }^{13}$.

Uma trajetória pode ser elaborada desde o descobrimento da patologia até a realização do seu tratamento, para compreender a vivência do portador. Nela existem momentos que se destacam como: o impacto do diagnóstico, que envolve diversos sintomas e sensações que levaram o indivíduo a procura de ajuda médica; o descobrimento de uma doença crônica que pode levar a sua morte; os limites da diálise, em que os pacientes que são submetidos se tornam reféns de uma máquina para sobreviver; as restrições alimentares e de hábitos rotineiros, que exigem uma mudança drástica dos seus comportamentos anteriores para aqueles suportados pela doença; e a liberdade do transplante, que permite a sensação de libertação da diálise, autonomia, esperança e fé ${ }^{14}$.

O diagnóstico da doença causa modificações no estilo de vida dessas pessoas e existem sentimentos que permeiam todo esse processo, como ansiedade, medo do desconhecido, tristeza, mudanças de humor e incertezas sobre o futuro. Existem também algumas experiências negativas que estas pessoas estão sujeitas a vivenciar como a morte dos companheiros de hemodiálise ou complicações no processo de saúde deles, antes ou após o início do tratamento ${ }^{10}$.

Alguns pacientes demonstram se sentir gratos pela diálise, pois esta proporcionou o prolongamento de suas vidas. Este sentimento de gratidão também se expressa como uma lealdade a um tratamento conhecido e que estão adaptados, fazendo com que o transplante se torne um procedimento menos conhecido e cheio de incertezas. Muitos pacientes criam vínculos na unidade de diálise e se sentem como parte de uma família, o que pode levar a não desejarem perder este laço de apoio ${ }^{15}$. Isso favorece para que estes pacientes permaneçam em diálise ao invés de buscarem pelo transplante renal, além das preocupações com o procedimento do transplante ${ }^{15-16}$.

Apesar da existência de efeitos negativos com relação à diálise, a autoavaliação de saúde em pacientes neste tratamento pode ser melhor que em pacientes ainda não dialíticos, devido aos sintomas relacionados à progressão da doença, os quais são aliviados pela terapia dialítica ${ }^{17}$.

Por outro lado, alguns pacientes que estão em tratamento dialítico apresentam o medo da morte, a ansiedade de um transplante, as incertezas com relação ao futuro e sentem-se limitados a algumas atividades do seu cotidiano. Entretanto, o recebimento de um órgão novo de um doador também acomete sensações negativas, embora se sintam felizes e temporariamente livres da terapia dialítica, a qual também Ihes causam sofrimento ${ }^{13}$. 
Para alguns destes pacientes, o recebimento de um órgão novo é visto como um presente divino, em que Deus os abençoou com um doador em suas vidas. A sua crença faz com que sensações positivas apareçam e o sentimento de gratidão por ser agraciado por uma nova oportunidade de retomar a antiga vida anterior a doença se estabelece ${ }^{13}$.

Apesar do anseio de uma vida mais semelhante à normal e saudável possível, permitida pelo transplante, os pacientes ainda apresentam medo com relação ao curso de sua doença e dúvidas com relação ao bom prognóstico após o transplante. Estas sensações negativas são reduzidas com as crenças, pois uma visão religiosa permite que estes coloquem a decisão do seu futuro atrelado a uma ordem divina, a qual alimenta a sua fé de que tudo dará certo. A busca pela religiosidade está atrelada à necessidade de uma proteção divina para enfrentar suas condições de adoecimento ${ }^{10}$.

Entretanto, apesar destes sentimentos, o receptor sabe que essa liberdade é apenas temporária, o que faz com que o medo e ansiedade retornem. O medo do enxerto falhar ou da idade estimada em relação a sobrevida do mesmo chegar, levam o paciente a sentir-se em uma contagem regressiva em que ele desconhece o tempo final, apenas sabe que este existe ${ }^{13}$.

O medo da morte e a relação de que o doador morreu e, por isso, ele pôde receber o seu órgão também são sentimentos e pensamentos que acometem esses pacientes. Além disso, nos casos em que o doador é vivo e membro da família, os sentimentos de insegurança com relação ao sucesso do transplante e os riscos que isto apresenta para o doador, conflitam as emoções de esperança por uma nova vida e a possibilidade de debilitar uma outra ${ }^{13}$.

Os pacientes com doença renal crônica podem apresentar sintomas depressivos independentemente do tipo de terapia renal substitutiva adotada. Estes sintomas são preditores de outras comorbidades inclusive do prognóstico da terapia. Sentimentos como desesperança também são encontrados nestes pacientes, uma vez que estes são acometidos por diversas incertezas sobre o futuro durante o seu processo de saúde e doença, e embora os impactos da doença afetem a vida do paciente, o seu contexto social também influencia no curso do seu tratamento ${ }^{18}$.
Além de sintomas depressivos e de desesperança, a ideação suicida também faz parte dos sentimentos vivenciados pelos portadores de DRCT, e em menor proporção que os sintomas depressivos como observado em um estudo comparativo de coorte transversal entre pacientes em tratamento de hemodiálise e pacientes transplantados. Neste estudo, não houve diferença entre a existência desses sintomas para ambas as terapias ${ }^{18}$.

Um estudo demonstrou a relação de sintomas de depressão e ansiedade em pacientes em terapia renal substitutiva. Observou-se a presença destes sintomas entre os pacientes e aqueles em tratamento de diálise apresentaram mais sintomas depressivos e de ansiedade que os pacientes transplantados, cerca de três vezes mais e 1,5 vezes mais, respectivamente ${ }^{19}$.

No mesmo estudo, a depressão esteve relacionada a condições mentais em que se encontravam os pacientes dialíticos e transplantados, já a ansiedade associou-se as condições mentais, físicas e à perda de acesso vascular nos últimos 12 meses nos pacientes em diálise apenas ${ }^{19}$.

São inúmeros sentimentos em que os pacientes renais em TRS podem apresentar durante o curso da doença e do seu tratamento, dentre eles estão sentimentos positivos e negativos os quais se intercalam de acordo com cada situação vivenciada pelo portador e escolha da TRS. Segundo Mato Grosso do Sul20, esses sentimentos acarretam consequências nos pacientes, os quais os tornam mais suscetíveis a conflitos e instabilidades.

Entretanto, apesar de se apresentarem comuns a algumas circunstâncias, eles também se divergem, demonstrando que a individualidade do paciente deve ser superior a quaisquer características emocionais previamente estudadas, as quais servem apenas como arcabouço teórico-científico para apresentação de possibilidades de intervenções terapêuticas. Para a Diretoria de Vigilância em Saúde de Mato Grosso do Sul${ }^{20}$ é papel do psicólogo, como integrante da equipe multidisciplinar na assistência ao portador renal crônico, motivar e orientar a pessoa desde o diagnóstico, nas unidades de tratamento e estender essa rede de apoio aos familiares/cuidadores. 
Existe uma insuficiência de materiais de referência nacional que auxiliem na atuação multiprofissional diante dos impactos psicológicos causados ao portador renal. Justificando a necessidade de documentos mais detalhados que possam ser usados como instrumento de apoio à equipe ${ }^{20}$.

\section{Expectativas relacionadas ao transplante}

Os pacientes em tratamento de hemodiálise criam expectativas com relação ao transplante, alimentando esperanças da chegada de um doador compatível e em um tempo mínimo possível, considerando a possibilidade de doadores vivos, o que poderia reduzir este tempo. $O$ transplante renal se apresenta como uma melhor solução para as limitações advindas da doença ${ }^{10}$.

Um estudo demonstrou que os pacientes idosos tinham expectativas com relação ao transplante renal e estes planejavam o futuro como se receber um rim proporcionasse a recuperação de uma vida. Apesar disso, também demonstravam estar cientes de que o transplante poderia não ter o sucesso que desejavam ${ }^{21}$.

Estar na lista de espera foi interpretado pelos pacientes como uma nova chance, alguns acreditavam que o receberiam e outros que talvez não, mas ainda assim, a possibilidade era enxergada como uma chance de adquirir uma vida menos limitada21.

Alguns pacientes demonstram estar conformados com o sentimento de viver esperando por um transplante. Apesar das expectativas de uma nova vida, eles entendiam que para isso poderia ser necessário um longo tempo de espera 21 .

Embora o transplante renal seja considerado a meIhor terapia para a doença renal terminal, para aqueles que apresentam indicação para a cirurgia, este exige um diversidade de cuidados para que o transplante seja bem-sucedido e promova uma melhor qualidade de vida. Isso leva alguns pacientes a não entrarem para a lista de espera e optarem por continuar em procedimentos dialíticos, como a hemodiálise, por se sentirem adaptados a esta rotina e pelo receio das complicações do transplante que podem levar à sua morte ${ }^{16}$.
Estes sentimentos e expectativas se consolidam quando um paciente presencia o retorno de um transplantado à hemodiálise, fazendo com que os receios de um insucesso com relação a um novo tratamento influenciem na tomada de decisão em não mudar de terapia ${ }^{16}$.

No estudo de Salter ${ }^{15}$, com relação ao sexo e idade, as mulheres e idosos apresentaram menos interesse em realizar o transplante, sendo sugerido pelos autores que os pacientes mais jovens têm uma consciência maior sobre suas limitações por serem mais ativos, além de que os pacientes mais velhos geralmente apresentam mais comorbidades com relação a idade que os mais jovens.

Esses resultados sugerem questionar quanto ao esclarecimento em relação aos riscos e benefícios da hemodiálise e do transplante renal, e reforçar a necessidade de que os profissionais de saúde, principalmente os enfermeiros, forneçam as informações necessárias e esclareçam o paciente sobre quaisquer dúvidas, garantindo que estes tenham compreensão sobre o seu tratamento de escolha e a importância devida das recomendações que este exige. Esta comunicação deve ser efetiva, utilizando-se de uma linguagem que seja acessível ao portador e com condutas que respeitem a sua cultura e seus valores. Estas medidas permitem que os pacientes tomem a sua decisão baseados em um conhecimento científico ${ }^{16}$.

Vale também reforçar que cada paciente apresenta um curso diferente em um mesmo tipo de tratamento, devido a sua correta adesão e as condições emocionais, físicas e sociais em que se encontra. Sendo assim, além das recomendações específicas de cada tratamento, existem aquelas específicas para cada paciente que variam de acordo com estes critérios e determinam o sucesso ou insucesso do tratamento ${ }^{16}$.

É fundamental que os profissionais de saúde conheçam a percepção do paciente renal crônico sobre o transplante para compreender melhor as suas necessidades individuais, bem como as motivações que levam estes pacientes a não ingressarem na lista de espera ${ }^{16}$. 
Segundo as Diretrizes Clínicas para o Cuidado ao Paciente com DRC22, o serviço de diálise deverá, em um prazo de noventa dias do início da diálise, apresentar ao paciente ou representante legal a opção de inscrição na lista de espera para transplante renal, se houver indicação desta terapêutica segundo o Regulamento Técnico do Sistema Nacional de Transplante. O paciente que não estiver em tratamento de diálise também poderá ser inscrito na lista para transplante, configurando a modalidade de transplante preemptivo, que é realizado antes de iniciar a TRS.

A atenção especializada em nefrologia deve: "garantir o acesso e assegurar a qualidade do processo de diálise, visando alcançar impacto positivo na sobrevida, na morbidade e na qualidade de vida, além de garantir equidade na inscrição em lista de espera para transplante" $\underline{20}$.

A correta orientação acerca das possibilidades terapêuticas, respeitando as condições biopsicossociais do paciente, deve ser priorizada e realizada de acordo com a sua situação clínica, pois esta orientação pode influenciar diretamente nas expectativas que $o$ portador terá com relação ao transplante.

\section{Transplante renal e a função do enxerto}

O transplante renal proporciona ao portador a libertação da diálise, identificada pelos pacientes como uma possibilidade de retorno a sua rotina habitual antes da doença, a liberdade para a realização de atividades sociais e de lazer, a não restrição alimentar e hídrica e a liberdade de não se preocupar com seu estado de saúde, trazendo a sensação de independência para ir e vir sem depender de outras pessoas para realizar o que precisa $a^{23}$.

Estes aspectos se configuraram como um impacto positivo na qualidade de vida dos pacientes, os quais sentem autonomia para suas atividades como viagens e estar com a família, além de voltarem a realizar atividades domésticas. Esta autonomia colabora para sensação de estar ativo, ser útil e capaz de desempenhar funções consideradas normais para um indivíduo saudável. A reaproximação com o cotidiano leva o paciente a sentir-se bem e melhora a sua qualidade de vida ${ }^{23}$.
Apesar de todas essas facilidades, existem dificuldades que os pacientes transplantados ainda precisam enfrentar como a rotina de cuidados que o transplante exige. A ideia de retorno para vida "normal" faz com que o paciente se decepcione com algumas limitações ainda presentes no transplante, como a restrição dietética e física, o uso de medicamentos imunossupressores (obedecer a horários e conviver com efeitos colaterais - o que pode dificultar a adesão), além dos cuidados necessários para preservação do enxerto, como proteger-se do frio e do contato com pessoas que apresentem alguma doença infecciosa ${ }^{23}$.

Com relação à função do enxerto, alguns autores apresentaram fatores que se relacionam a função retardada do enxerto (FRE) em pacientes transplantados renais, que leva em consideração a necessidade de diálise na primeira semana pós-cirurgia. Dentre estes fatores estão: doador falecido, anestesia combinada, duração de diálise pré-transplante > que 60 meses, regime de fluidos restritivos e uso de Basiliximab ${ }^{24}$.

O fator "doador falecido" foi relacionado ao tempo de isquemia fria, bem como a dificuldade em manter uma estabilidade hemodinâmica do doador e rins de critérios expandidos. A anestesia combinada apresentou risco três vezes maior de disfunção do enxerto e os autores sugerem que a redução dos níveis de catecolaminas e a vasodilatação após o bloqueio neuroaxial diminui a perfusão do enxerto, podendo levar a essa disfunção. O Basiliximab, um dos medicamentos imunossupressores utilizados no período pós-transplante, libera citocinas e induz a nefrotoxidade ${ }^{24}$.

A readmissão hospitalar precoce (RHP) após a realização do transplante, que consiste na internação hospitalar em menos de 30 dias pós-cirurgia, aumenta o risco de perda enxerto, principalmente em pacientes idosos 25 .

Outros fatores que dificultam a experiência do paciente são: prescrições médicas erradas e o risco da falta de medicamentos suficientes disponibilizados pelo sistema de saúde, o que dificulta o acesso a medicação, levando os pacientes a se preocuparem com relação a sua função renal e a preservação do enxerto, além de necessitarem adotar medidas alternativas para adquirir os medicamentos ${ }^{23}$. 
Apesar das facilidades proporcionadas pelos transplantes e estas se sobressaírem às dificuldades, como demonstrado pelo estudo, a idealização de cura é interrompida quando os pacientes se deparam com as limitações deste tratamento. Ressalta-se a importância de lembrar que estas facilidades e dificuldades são vivenciadas de formas diferentes pelos pacientes, o que se faz necessário que os profissionais de saúde, em destaque a enfermagem, promovam ações voltadas para as particularidades e contexto de cada paciente transplantado $\underline{23}$.

O transplante renal é visto como uma terapêutica de "cura" por alguns pacientes antes de receber o enxerto, os mesmos acabam encontrando dificuldades ao longo do tratamento. A equipe multiprofissional deve adotar medidas voltadas para a melhoria da qualidade de vida do portador, como o esclarecimento acerca desta terapêutica e a possibilidade de rejeição do enxerto. Estas medidas irão impactar no sucesso do transplante minimizando algumas dificuldades encontradas, como a interrupção tardia da idealização de cura e a adesão a medicação.

Segundo o Protocolo Clínico e Diretrizes Terapêuticas - Portaria no 666/201226, antes da realização do transplante o médico deverá garantir orientações e esclarecimentos relacionados à terapia medicamentosa de imunossupressão e a necessidade da adesão para diminuir a possibilidade de rejeição do enxerto, com a assinatura do Termo de Esclarecimento e Responsabilidade pelo médico solicitante e pelo paciente ou seu responsável.

\section{Fatores que interferem na qualidade de vida durante a terapia renal substitutiva}

Um estudo de coorte prospectivo com pacientes em diálise e transplantados renais mostrou que a QVRS medida na diálise, mais especificamente relacionada aos aspectos físicos do paciente, pode prever a sobrevida do paciente ex-dialítico após o transplante renal, e que pacientes com uma melhor função física referida apresentam menor comorbidade. Esta afirmação sugere que os pacientes classificados com uma função física diminuída na diálise podem apresentar riscos pós transplante renal, reforçando a ideia de que a atividade física deve ser recomendada e estimulada em pacientes renais de acordo com as suas limitações ${ }^{27}$.
A concepção acerca da qualidade de vida é influenciada por diversos fatores, dentre eles a compreensão do paciente acerca do seu processo de adoecimento, a sua cultura e as experiências vivenciadas durante este processo. Para os pacientes transplantados que obtém o enxerto funcionante, a qualidade de vida é aumentada em relação à hemodiálise, devido aos grandes benefícios que este tratamento proporciona como a sensação de reconquista da sua saúde, liberdade e autonomia sobre sua vida ${ }^{14}$.

Os impactos causados durante a trajetória do paciente podem levar o indivíduo a apresentar manifestações psíquicas negativas em relação ao seu processo de adoecimento, podendo acarretar sentimentos depressivos. Ressalta-se a importância de um acompanhamento psicológico desde o diagnóstico para evitar que estes impactos inferiram ainda mais na qualidade de vida destes pacientes ${ }^{14}$.

No estudo de Lai ${ }^{28}$ foi observado que os pacientes em tratamento de hemodiálise administram melhor suas emoções quando comparados aos pacientes em terapia conservadora, o que pode estar relacionado com o não envolvimento emocional dos pacientes nos estágios iniciais da doença, para evitar sensações negativas como os sentimentos depressivos. Em contrapartida, os pacientes hemodialíticos são coagidos a vivenciar as emoções devido ao impacto causado pelo tratamento que influencia diretamente no seu estilo de vida. Reforçando-se a necessidade de um acompanhamento multiprofissional voltados para as necessidades de cada paciente e sua terapia.

A qualidade de sono é fundamental para um bom curso da terapia e melhoria da qualidade de vida, visto que os pacientes que dormem mal apresentam mais sintomas depressivos que aqueles que dormem bem, e isso não difere com relação ao tipo de terapia (diálise e transplante renal). A qualidade de sono foi observada, em um dos estudos, como melhor após a realização do transplante renal, isso pode estar associado a melhoria dos sintomas urêmicos. Alguns pacientes obtiveram uma piora desta qualidade e o uso de imunossupressores podem contribuir para este resultado, além das preocupações com o enxerto. Entretanto, o número de pacientes que apresentaram uma melhora significativa se manteve maior que aqueles que apresentaram uma piora relevante ${ }^{29}$. 
Os pacientes submetidos à diálise peritoneal, apesar de terem uma maior autonomia para regime dietético, podem apresentar, devido a isto, uma menor adesão ao tratamento medicamentoso. Essa dificuldade de adesão pode estar associada a questões psicológicas que afetam a sua experiência, como o estresse ${ }^{28}$.

Os níveis de hemoglobina dos pacientes em hemodiálise e diálise peritoneal são inferiores aos dos pacientes em tratamento conservador e transplante renal. Nos pacientes em diálise peritoneal, os níveis de fósforo sérico são consideravelmente maiores, e ambos os valores da hemoglobina e fósforo para os pacientes dialisados podem estar associados também a questões psicológicas, o que torna imprescindível um acompanhamento psicológico direcionado desde os estágios iniciais da doença. A diminuição do fósforo sérico é fundamental para o tratamento do paciente renal, uma vez que esta alteração está relacionada com a progressão da lesão renal e doenças cardiovasculares ${ }^{28}$.

Alguns autores sugerem que pacientes dialíticos com uma saúde mental debilitada antes do transplante podem levar a diminuição da função do enxerto renal, devido os sintomas depressivos estarem relacionados ao risco de não adesão ao tratamento medicamentoso imunossupressor, o qual é fundamental para o bom funcionamento do enxerto, e, portanto, para uma melhor qualidade de vida ${ }^{27}$.

Em um estudo transversal ${ }^{19}$ com participantes de uma coorte de 205 pacientes de dez serviços de diálise públicos em Belo Horizonte, estabelecida em 2006, com o objetivo de investigar as prevalências de depressão e ansiedade entre os pacientes em terapia renal substitutiva, foi observado que a pior qualidade de vida dos pacientes esteve associada aos sentimentos depressivos em pacientes transplantados. Nos pacientes em diálise os sintomas de ansiedade associaram-se à pior qualidade de vida. Também relacionado à ansiedade estiveram a dor corporal e uma menor realização de atividades recreativas, aumentando a gravidade dos sintomas.

Outro estudo com afro-americanos demonstrou que pacientes mais velhos tendem a ser mais positivos com relação a experiência de diálise quando têm o apoio de amigos e familiares. Da mesma forma, pacientes mais jovens tendem a ter experiências menos positivas quando não têm o apoio das famílias e amigos. O apoio familiar é fundamental para o enfrentamento da doença ${ }^{15}$.
Com relação à função cognitiva, o declínio desta foi observado em pacientes em tratamento de hemodiálise regular em comparação a pacientes transplantados e pacientes com boas condições de saúde, embora os autores relatem que o estudo, por ser transversal, impede o estabelecimento de relação causal entre hemodiálise e comprometimento cognitivo, expressando a necessidade de estudos longitudinais que permitam justificar este achado. O transplante renal foi observado como terapia que contribui para preservação cognitiva dos portadores de doença renal terminal ${ }^{30}$.

Dois estudos, sendo um de origem nacional com pacientes transplantados e outro internacional com pacientes em diálise, demonstraram uma falta de informação com relação à terapêutica de transplante. Os profissionais de saúde não forneciam informações adequadas sobre o tratamento dialítico e sobre a possibilidade de transplante, mesmo quando alguns pacientes solicitavam ter conhecimento. Os depoimentos dos pacientes de ambos os estudos demonstraram que devido às dificuldades na relação com os profissionais, estes apresentaram inúmeras preocupações com relação ao transplante renal, incluindo o medo da cirurgia, da ingestão de muitos medicamentos, do insucesso do transplante, e, nos pacientes mais velhos, a idade avançada ${ }^{15,23}$.

Os profissionais que acompanham o paciente renal crônico devem estar atentos a estes impactos para prestarem o apoio necessário a estes pacientes, bem como fornecer informações suficientes e adequadas, ressaltando a importância da adesão e do autocuidado, para que o seu tratamento seja realizado da meIhor maneira possível e a qualidade de vida não seja ainda mais impactada ${ }^{10}$.

O vínculo efetivo entre o profissional e paciente com uma comunicação efetiva, amenizando os sentimentos de ansiedade, expectativas e medos, é um caminho necessário para a melhoria do seu processo de saúde, contribuindo para a melhoria da qualidade de vida do paciente. A enfermagem, por estar em constate contato direto com o paciente, necessita se adequar as necessidades desta doença e as peculiaridades de cada indivíduo para uma assistência humanizada e um cuidado holístico e efetivo, que contribuam com o bem-estar do paciente e proporcione uma experiência mais positiva, consequentemente uma melhor qualidade de vida ${ }^{10}$. 
Esta categoria, com alguns impactos físicos e psicológicos das terapias renais substitutivas que interferem na qualidade de vida dos pacientes renais terminais, demonstra que o caminho para uma terapêutica que alcance a melhor qualidade de vida do paciente necessita de uma assistência multidisciplinar comprometida em garantir o entendimento do mesmo sobre o seu processo de adoecimento, assim como em apresentar terapias que abarquem as suas necessidades de maneira holística.

\section{Conclusão}

Os estudos demonstraram que existem vários fatores relacionados a terapia renal substitutiva que interferem na qualidade de vida dos pacientes renais terminais, dentre eles estão os impactos físicos causados por cada tipo de tratamento, os impactos psicológicos causados por todos os sentimentos e expectativas vivenciadas durante este processo e a qualidade da assistência prestada, evidenciados à partir das quatro categorias apresentadas.

Dentre os impactos físicos estão às limitações de atividades cotidianas, restrições alimentares e hídricas, adoção de rotinas, adesão de medicamentos e comportamentos para se adequar ao tratamento, além dos distúrbios eletrolíticos causados pela doença e às vezes não controlados pelo tratamento. Com relação aos impactos psicológicos estão todos os sentimentos e sensações, como o medo da morte e ansiedade por um tratamento que proporcione uma vida normal, sentimentos depressivos advindos da insegurança e incerteza sobre o futuro, além das variadas expectativas com relação ao transplante, as quais podem estar associados à falta de informações adequadas sobre o seu processo de saúde e tratamento.

O apoio psicológico aos pacientes foi reconhecido como necessário desde o diagnóstico, para amenizar os sentimentos advindos dos impactos causados pela doença e pelo tratamento. Além disso, o vínculo entre profissionais e pacientes deve ser estabelecido da melhor forma possível com uma comunicação efetiva e adequada as condições culturais, sociais e religiosas de cada indivíduo, bem como uma assistência que respeite todas as suas limitações. O fornecimento de informações necessárias sobre o tratamento e a garantia da compreensão do paciente sobre essas informações é fundamental. A assistência de enfermagem se destacou como a profissão que detém papel essencial no estabelecimento deste vínculo.

\section{Limitações do estudo}

Não houve limitações para a realização do estudo apresentado.

\section{Contribuições das autoras}

Pereira LTC participou da concepção, delineamento, busca, interpretação e análise dos dados da pesquisa, interpretação dos resultados e redação do artigo científico. Ferreira MMM participou como orientadora e revisora do artigo científico.

\section{Conflitos de interesses}

Nenhum conflito financeiro, legal ou político envolvendo terceiros (governo, empresas e fundações privadas, etc.) foi declarado para nenhum aspecto do trabalho submetido (incluindo, mas não se limitando a subvenções e financiamentos, participação em conselho consultivo, desenho de estudo, preparação de manuscrito, análise estatística, etc.).

\section{Referências}

1. Kirsztajn GM. Doença renal crônica: diagnóstico e prevenção. Sociedade Brasileira de Nefrologia [Internet]. 2020. [acesso em 2020 abr 26]. Disponível em: https://www.sbn.org.br/noticias/ single/news/doenca-renal-cronica-diagnostico-e-prevencao/

2. Ministério da Saúde. 14/3 - Dia mundial do rim 2019: saúde dos rins para todos. Biblioteca Virtual em Saúde [Internet]. 2019. [acesso em 2020 jul 11]. Disponível em: https://bvsms.saude.gov. br/ultimas-noticias/2913-14-3-dia-mundial-do-rim-2019-saudedos-rins-para-todos

3. Neves PDMM, Sesso RCC, Thomé FS, Lugon JR, Nasicmento MM. Censo Brasileiro de Diálise: análise de dados da década 20092018. Braz J Nephrol. 2020;42(2):191-200. doi: 10.1590/2175-8239jbn-2019-0234
4. Associação Brasileira de Transplantes. Registro Brasileiro de Transplantes. Dimensionamento do Transplantes no Brasil e em cada Estado (2012-2019). [Internet]. 2019. [acesso em 2020 jul 11]. Disponível em: http://www.abto.org.br/abtov03/Upload/file/ RBT/2019/RBT-2019-leitura.pdf 
5. Associação Brasileira de Transplantes. Registro Brasileiro de Transplantes. Dados numéricos da doação de órgãos e transplantes realizados por estado e instituição no período: janeiro / março - 2020. [Internet]. 2020. [acesso em 2020 jul 11]. Disponível em: http://www.abto.org.br/abtov03/Upload/file/ RBT/2020/RBT-2020-1 trim-leitura.pdf

6. Gonçalves FA, Dalosso IF, Borba JMC, Bucaneve J, Valerio NMP, Okamoto CT et al . Qualidade de vida de pacientes renais crônicos em hemodiálise ou diálise peritoneal: estudo comparativo em um serviço de referência de Curitiba - PR. J Bras Nefrol. 2015;37(4): 467-474. doi: 10.5935/0101-2800.20150074

7. Santos RSS, Sardinha AHL. Qualidade de vida de pacientes com doença renal crônica. Enferm Foco. 2018;2(9):61-66.

8. Noronha DD, Martins AMEBL, Dias DS, Silveira MF, Paula $A M B$, Haikal DSA. Qualidade de vida relacionada à saúde entre adultos e fatores associados: um estudo de base populacional. Ciênc Saúde Coletiva. 2016;21(2):463-474. doi: 10.1590/1413$\underline{81232015212.01102015}$

9. Cruz DSM, Collet N, Nóbrega VM. Qualidade de vida relacionada à saúde de adolescentes com dm1 - revisão integrativa. Ciênc Saúde Coletiva. 2018;23(3):973-989. doi: 10.1590/1413$\underline{81232018233.08002016}$

10. Farias MS, Maia ICG, Ferreira GSM, Pinto JR, Ferreira FIS. Sentimentos de pessoas em hemodiálise que esperam por um transplante renal. Rev Bras Ciênc Saúde. 2018;22(4): 357-362. doi: 10.22478/ufpb.2317-6032.2018v22n4.28164

11. Mendes KDS, Silveira RCCP, Galvão CM. Revisão integrativa: método de pesquisa para a incorporação de evidências na saúde e na enfermagem. Texto Contexto Enferm. 2008;17(4):758-64. doi: 10.1590/S0104-07072008000400018

12. Ingelfinger JR, Schaefer F, Kalantar-Zadeh K. Evitando o legado da doença renal - Foco na infância. J Bras Nefrol. 2016;38(1):2-8. doi: $10.5935 / 0101-2800.20160002$

13. Ramírez CA. Aprender a vivir con un órgano trasplantado: Learning to live with a transplantedorgan. Rev Cienc Cuidad. 2019;16(3):95-104. doi: $10.22463 / 17949831.1596$

14. Santos LF, Prado BC, Castro FPS, Brito RF, Maciel SC, Avelar TC. Qualidade de Vida em Transplantados Renais. Psico-USF. 2018;23(1):163-172. doi: 10.1590/1413-82712018230114

15. Salter ML, Kumar K, Law AH, Gupta N, Marks K, Balhara K et al. Percepções sobre hemodiálise e transplante em adultos afroamericanos com doença renal terminal: inferências de grupos focais. BMC Nephrol. 2015;16:49. doi: 10.1186/s12882-015-0045-1

16. Pauletto MR, Beuter M, Timm AMB, Santos NO, Roso CC, Jacobi CS. Transplante renal: percepção de pacientes em hemodiálise fora da lista de espera. Revista de Enfermagem da UFSM.

2016;6(2):154-163. doi: 10.5902/2179769220619
17. Grove BE, Schougaard LM, Hjollund NH, Ivarsen P. Self-rated health, quality of life and appetite as predictors of initiation of dialysis and mortality in patients with chronic kidney disease stages 4-5: a prospective cohort study. BMC Res Notes. 2018;11(1):371. doi: 10.1186/s13104-018-3472-9

18. Andrade SV, Sesso R, Diniz DHMP. Desesperança, ideação suicida e depressão em pacientes renais crônicos em tratamento por hemodiálise ou transplante. J Bras Nefrol. 2015;37(1):55-63. doi: $\underline{10.5935 / 0101-2800.20150009}$

19. Brito DCS, Machado EL, Reis IA, Carmo LPF, Cherchiglia ML. Depression and anxiety among patients undergoing dialysis and kidney transplantation: a cross-sectional study. São Paulo Med J. 2019;137(2):137-47. doi: 10.1590/1516-3180.2018.0272280119

20. Secretaria de Estado de Saúde de Mato Grosso do Sul. Diretoria de Vigilância em Saúde. Coordenadoria de Vigilância Sanitária. Atenção transdisciplinar ao renal crônico: manual para abordagem de pacientes em tratamento hemodialítico. [Internet]. 2011. [acesso em 2020 jul 10]. Disponível em: http://www. as.saude.ms.gov.br/wp-content/uploads/2016/08/manual_renalx1a.pdf

21. Lønning K, Midtvedt K, Heldal K, Andersen MH. Older kidney transplantation candidates' expectations of improvement in life and health following kidney transplantation: semistructured interviews with enlisted dialysis patients aged 65 years and older. BMJ Open. 2018;8(6):e021275. doi: 10.1136/bmjopen-2017-021275

22. Ministério da Saúde. Secretaria de Atenção à Saúde. Departamento de Atenção Especializada e Temática. Diretrizes Clínicas para o Cuidado ao paciente com Doença Renal Crônica - DRC no Sistema Único de Saúde/ Ministério da Saúde. Brasília: Ministério da Saúde; 2014.

23. Santos BP, Viegas AC, Feijó AM, Lise F, Schwartz E. Foi/não foi tudo o que pensava: facilidades e dificuldades após o transplante renal. Rev Gaúcha Enferm. 2016;37(3). doi: 10.1590/1983$\underline{1447.2016 .03 .60135}$

24. Freitas MHB, Lima LC, Couceiro TCM, Silva WB, Andrade $J M$, Freitas MHB. Fatores perioperatórios associados à função retardada do enxerto em pacientes transplantados renais. J Bras Nefrol. 2018;40(4):360-365. doi: 10.1590/2175-8239-jbn-2018-0020

25. Haugen CE, King EA, Bae S, Bowring MG, Holscher CM, Garonzik-Wang J et al. Early Hospital Readmission in Older and Younger Kidney Transplant Recipients. Am J Nephrol. 2018;48(4):235-241. doi: 10.1159/000492338

26. Brasil. Ministério da Saúde. Portaria n 666, de 17 de julho de 2012. Aprova o Protocolo Clínico e Diretrizes Terapêuticas Imunossupressão no Transplante Renal. Diário Oficial da União. 2012 jul. 17. 
27. Lippe NVD, Waldum-Grevbo B, Reisæter AV, Os I. Is HRQOL in dialysis associated with patient survival or graft function after kidney transplantation? BMC Nephrol. 2016;17:94. doi: 10.1186/ s12882-016-0316-5

28. Lai C, Aceto P, Luciani M, Fazzari E, Cesari V, Luciano $S$ et al. Emotional management and biological markers of dietetic regimen in chronic kidney disease patients. Ren Fail. 2017;39(1):173-178. doi: 10.1080/0886022X.2016.1256312
29. Brekke FB, Waldum-Grevbo B, Lippe NVD, Os I. The effect of renal transplantation on quality of sleep in former dialysis patients. Transpl Int. 2017;30(1):49-56. doi: 10.1111/tri.12866

30. Anwar W, Ezzat H, Mohab A. Comparative study of impact of hemodialysis and renal transplantation on cognitive functions in ESRD patients. Nefrologia. 2015;35(6):567-571. doi: 10.1016/j. nefro.2015.07.004 